\title{
PROBLEMA DA MOCHILA 0/1 PARA DETERMINAR O PADRÃO DE ATENDIMENTO DOS PORTOS
}

\author{
João do Nascimento Farias Júnior \\ Universidade Federal do Amazonas - UFAM \\ joaofariasjunior92@gmail.com \\ Ricardo Alexandre Deiró Santos \\ Instituto de Pesquisas em Transportes - INTRA \\ ralexandredeiro@gmail.com \\ Larissa de Andrade Silva \\ Instituto de Pesquisas em Transportes - INTRA \\ larissa.andsilva@gmail.com \\ José Teixeira de Araújo Neto Santos \\ Instituto de Pesquisas em Transportes - INTRA \\ teixeira.santos@intra.org.br \\ Waltair Vieira Machado \\ Universidade Federal do Amazonas - UFAM \\ waltair_machado@yahoo.com.br
}

\begin{abstract}
Resumo
O trabalho tem como objetivo selecionar os itens de padrão de atendimento que compõem um porto, através do uso do "Problema da Mochila 0/1. A modelagem do problema portuário foi feita adotando as funções custo e importância do padrão de atendimento, como variáveis que determinam os itens que irão compor a "mochila" (o porto) para que estes possam operar de maneira satisfatória. O modelo avalia a importância do item e seu custo de implantação no porto, obedecendo as restrições de custo imposta pelo usuário, desejando-se sempre encher a mochila com o maior valor possível de itens sem exceder sua capacidade. Os resultados obtidos pelo "Problema da Mochila" mostram que os cenários obtidos são mais eficientes que os atuais e podem ser utilizados com referência para que portos considerados ineficientes e melhorem seus desempenhos no padrão de atendimento.
\end{abstract}

Palavras-Chaves: Problema da Mochila 0/1; Porto; desempenho.

\begin{abstract}
The study aims to select the standard of care items that make up a port through the use of the 0/1 knapsack problem. The modeling of the port problem was made by adopting the cost functions and importance of standard of care, as variables that determine the items that will make up the "pack" (the port) so that they can operate satisfactorily. The model assesses the importance of the item and its deployment cost in port, obeying the cost constraints imposed by the user, wishing to always fill the backpack with the highest possible value items without exceeding its capacity. The results of the $0 / 1$ knapsack problem show that the obtained scenarios are more efficient than the current and can be used with reference to ports considered inefficient and improve their performance in the standard of care.
\end{abstract}

Keywords: 0/1 knapsack problem; Port; performance. 


\section{INTRODUÇÃO}

O modal aquaviário é o meio de transporte de pessoas e mercadorias mais importante na região amazônica, interligando comunidades e polos de produção, comercialização e consumo situados nas proximidades da vasta malha hidroviária. A dificuldade para desenvolver um eficiente transporte aquaviário é de diversas ordens, reflexo dos baixos investimentos no setor ao longo dos anos, resultando numa precária infraestrutura das instalações portuárias da região [4].

O país conta com 37 portos públicos, entre marítimos e fluviais, com uma infraestrutura que apresenta problemas tais como: acesso aos portos, à escassez de equipamentos de terra, falta de dragagem e manutenção. Com isso, o custo de operação portuária é elevado no país quando comparado aos demais portos do mundo o que faz com que operem abaixo de sua capacidade total [6].

A região amazônica apresenta peculiaridades operacionais, com uma dispersão de instalações portuárias e predominância de práticas informais marcadas pela cultura local e suas tradições, que contribuem para a escassez de informações quantitativas e qualitativas a respeito do padrão de infraestrutura portuária para o transporte fluvial de passageiros e cargas [4].

Essas práticas informais que envolvem transporte de cargas e passageiros acontecem em embarcações que atracam em portos e ou terminais hidroviários que não apresentam compatibilização entre seus projetos arquitetônicos de portos com os estudos de planejamento de transportes realizados [11].

A falta de infraestrutura adequada, as deficiências de projeto e a não integração do sistema hidroviário com os demais modos de transporte, na maioria dos portos da região, são responsáveis por grande parte dos problemas operacionais ocorridos e pela não confiabilidade dos usuários do sistema [11].

O presente artigo tem como objetivo geral apresentar uma metodologia para selecionar os itens que compõem um porto, para que através do planejamento portuário, a região seja capaz de oferecer serviços portuários que atendam a expansão da demanda com bons níveis de qualidade para o transporte misto.

O problema selecionar os itens para o porto foi tratado com o Problema da Mochila 0/1, adotando as funções custo e importância, como variáveis que determinam os itens que irão compor a "mochila" (o porto).

O resultado da aplicação da metodologia apresentada pode proporcionar uma melhoria do transporte fluvial na Amazônia. A aplicação do modelo proposto oferece um diagnóstico de onde deve ser melhorado, influencia no planejamento das políticas públicas, na regulamentação, qualificação e especialização do setor, além de fornecer alinhamento conceitual aos usuários.

O trabalho foi dividido em 4 (quatro) seções; a primeira seção aborda conceitos de transporte aquaviário, portos e sua importância para a região e o resultado da pesquisa desenvolvida pelo INTRA - Instituto de Pesquisa em Transporte sobre o relatório executivo desenvolvido pela ANTAQ. A segunda seção traz a fundamentação teórica do problema da mochila, na qual é apresentado o modelo matemático para o problema. Na terceira seção é apresentada a aplicação do problema e, na seção posterior, os resultados e discussão gerados pela aplicação do modelo.

\section{PORTO}

A atividade portuária é algo mais complexa e não se limita apenas à sua estrutura física. O porto possui a estrutura física, que compreende a infraestrutura aquaviária 
disponível, as instalações de terra e os equipamentos utilizados para manuseio, carga e descarga das mercadorias, necessitam ainda de organizações, equipamentos e sistemas que possibilitem a melhor rapidez, segurança e menor custo para os deslocamentos das mercadorias e pessoas. Esse conjunto de atividades abrange atualmente o que se chama de logística. [8]

Para conceituar um porto, é necessário compreender o que é o transporte aquaviário e identificar três aspectos que fazem parte desse modal de transporte: as infraestruturas aquaviárias, portuária e terrestre. [9]

A infraestrutura aquaviária é composta pelos canais de acesso aos portos, bacias de evolução, quebra-mares, hidrovias e berços de atracação. Os equipamentos para movimentação e armazenagem de mercadorias, tais como guindastes, esteiras e armazéns, são conhecidos como superestrutura portuária. Já infraestrutura portuária é composta pela estrutura fixa sobre os quais é realizada a movimentação de cargas entre os navios e os modais terrestres. Finalmente, a infraestrutura terrestre permite o transporte de bens entre os navios e os limites da área do porto, por meio não somente de vias ferroviárias e rodoviárias, dutos e correias transportadoras, mas também dos pátios dos terminais de embarque e desembarque de cargas e passageiros, e dos pátios das áreas de armazenagem. [9]

Foram classificados os portos em três tipos: quanto à localização, quanto à infraestrutura e quanto à atividade [7]. A localização é o espaço em que se implanta a estrutura portuária; A decisão por este local é determinada por diversas condições, principalmente, pela integração com modos de transporte terrestre [14]. Rodrigues [16] afirma que a localização do porto pode influenciar diretamente nos custos de transporte.

Os portos são formados, em geral, por um conjunto de elementos essenciais a sua operação. São compreendidos pelas instalações portuárias, quais sejam ancoradouros, docas, cais, pontes e píeres de atracação e acostagem, terrenos, armazéns, edificações e vias de circulação interna, bem como pela infraestrutura de proteção e acesso aquaviário ao porto, tais como guias-correntes, quebra-mares, eclusas, canais, bacias de evolução e áreas de fundeio que devam ser mantidas pela Administração do Porto (Lei 8.630/93) [3].

Há ainda o conceito de porto organizado, que "é aquele construído e aparelhado para atender às necessidades da navegação, da movimentação e da armazenagem de mercadorias, concedido ou explorado pela União, cujo tráfego e operações estejam sob a jurisdição de uma autoridade portuária" [3].

Existem também as instalações públicas de pequeno porte (IP4) que outorga de autorização somente a Estados e Municípios. Ainda que exploradas por estados ou municípios, são consideradas instalações portuárias federais. Operam exclusivamente com embarcações classificadas ou certificadas para a navegação interior. Poderá realizar operações de embarque e desembarque de passageiros, bem como a movimentação e armazenagem de mercadorias, ou ambas. Instalações de uso público, com possibilidade de transferência da atividade à iniciativa privada (arrendamento), mediante regular processo licitatório [1].

Finaliza-se a abordagem de conceitual de portos, com a definição e a finalidade das Estações de Transbordo de Carga (ETC), que tem grande capacidade de atingir distantes mercados através do Longo Curso e Cabotagem. A ETC surgiu da necessidade de regularização dos pontos de transbordo de cargas, localizados ao longo das hidrovias, em especial na bacia amazônica e hidrovia Tietê-Paraná. Terminais dessa classificação são utilizados, exclusivamente, para operação de transbordo de cargas destinadas ou provenientes da navegação interior. Portanto, ETC e IP4 apresentam-se como instrumentos de desenvolvimento regional. [2]. 


\subsection{PADRÃO DE ATENDIMENTO DOS PORTOS}

A ANTAQ [4] define os padrões de atendimento para as embarcações e instalações portuárias operarem de maneira satisfatória. Tal relatório executivo foi desenvolvido com o objetivo de investigar e orientar sobre a maioria dos problemas que envolvem o transporte hidroviário de passageiros na Amazônia, esperando servir de subsidio para melhores práticas regulatórias e ações efetivas no transporte aquaviário misto.

A avaliação dos terminais feita no estudo citado mostra os itens necessários para o atendimento dos usuários segundo critérios técnicos e operacionais. Entretanto, não foi definido neste estudo um padrão mínimo necessário para o funcionamento adequado de um terminal portuário.

O nível de atendimento de acordo com os critérios da ANTAQ é caracterizado como: elevado, se forem atendidos $70 \%$ a $100 \%$ dos critérios de padrão de atendimento; médio, se forem atendidos 50\% a 69\%; e baixo, se forem atendidos $0 \%$ a $49 \%$ dos critérios. [4]

Seguindo os critérios de padrão de atendimento citado, realizou-se uma pesquisa qualitativa objetivando apresentar os itens mínimos necessários para o funcionamento adequado de um terminal de passageiro. Trinta (30) terminais de passageiros foram submetidos à avaliação da ANTAQ no Amazonas, que obtiveram classificação baixa. Foi observado que existe uma discrepância grande entre os terminais do Estado, justificado pelo desequilíbrio socioeconômico no território do Amazonas. O terminal do Roadway foi o melhor classificado, segundo a ANTAQ, com 70\% de observância dos itens pesquisados, enquanto o Porto São Raimundo aproximadamente 3\%. [4]

No Amazonas, a pesquisa foi marcada pela ausência, principalmente, de critérios relacionados à operacionalidade. Requisitos de atendimento, como posto de atendimento médico, área de circulação com sinalização e telefones públicos apresentaram percentuais muitos baixos, apesar de se tratar de investimentos relativamente pequenos. Outro aspecto que deve ser colocado, ainda na demanda de operação, é a questão dos berços de atracação adequados, que apesar de ter importância primária, apresenta percentual baixo nos terminais do Estado. [4]

Os percentuais de atendimento global dos critérios analisados demonstraram um desempenho ruim para os terminais do Amazonas. Tal que, $80 \%$ deles obtiveram avaliação inferior a 30\% (baixo). Apenas 4 (quatro) terminais obtiveram avaliação superior a 50\% (médio ou alto), são eles: Roadway, Ceasa, Tabatinga e Humaitá. [4]

Dos terminais avaliados no estado do Amazonas, $87 \%$ deles apresentam baixo padrão de atendimento, $10 \%$ médio e apenas $3 \%$ apresentam um padrão elevado. Logo, a pesquisa reforça a necessidade de melhorias nos terminais do Estado com o intuito de atingir um padrão adequado. [4]

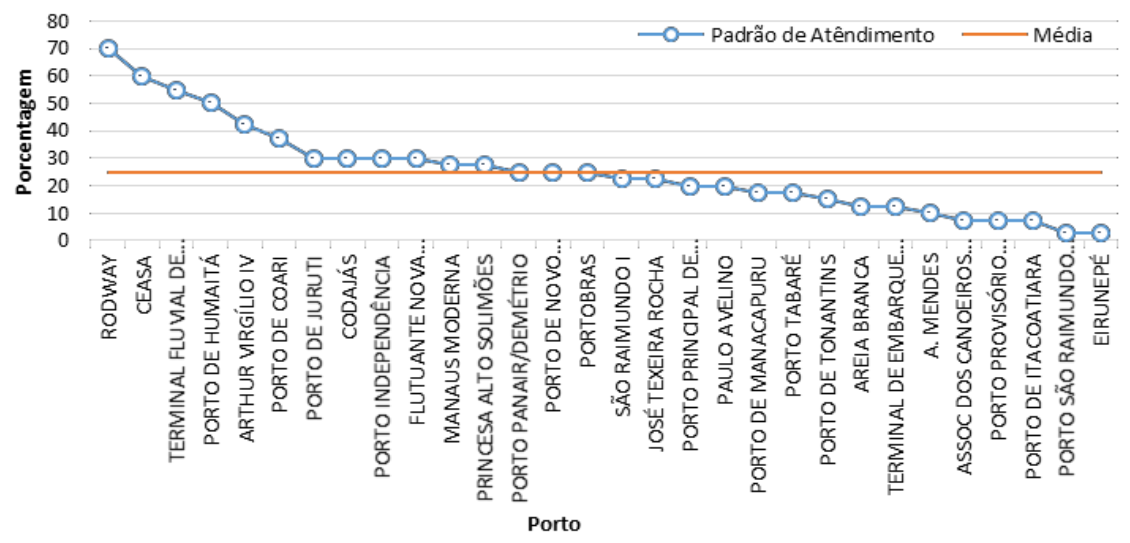

Figura 1 - Padrão de Atendimento nos portos de Manaus

Fonte: [1] 
Portanto, os problemas de infraestrutura e operacionalização interferem no bom funcionamento dos terminais portuários de Manaus. Adequar os portos a esses critérios tornaria o transporte mais eficiente no ponto de vista econômico e mais confortável para os usuários, diminuindo os riscos que os passageiros estão sujeitos ao utilizar este transporte.

\section{PROBLEMA DA MOCHILA}

Como Problemas de otimização combinatória que possui conjunto de soluçõesviáveis finito e discreto [11], o problema da mochila (KnapSack Problem) é um exemplo que possui característica $N P$-completo [17], no qual, em teorias de complexidade computacional, NP (nondeterministic polynomial time) se refere a um conjunto de problemas de decisão cuja a solução não pode ser reduzida a tempo polinomial, e NP-Completo um subconjunto de NP mais difíceis.

Definição de Knapsack problem seria dado um conjunto de itens, cada um com um peso e um valor, determinar o número de itens a serem incluídos de modo que o peso total é menor do que ou igual a um determinado limite e o valor total é o maior possível [17]. Foi estudado exaustivamente, por ter aplicações na indústria (nas áreas de logística, transporte e produção), mas primariamente por ser um problema de alocação.

Nesse artigo, foi usada uma das variações do problema de 1972, chamado 0/1 Knapsack Problem (Problema da Mochila 0/1), de característica NP-difícil. A mochila booleana inteira define as variáveis em $\{0,1\}$ para que se maximizem a função-objetivo sob as restrições do problema em questão. Ou seja, uma variável só pode ser escolhida no máximo uma vez, portanto podendo estar na mochila (1) ou não (0), mas ainda seguindo a restrições do problema principal. [12] [13]

\subsection{FORMULAÇÃO DO PROBLEMA DA MOCHILA 0/1}

O problema pode ser declarado da seguinte forma [15]: são dados $n$ itens para serem colocados em uma mochila de capacidade c. Cada item $j$ tem um valor $p_{j}$ e peso $w_{j}$ associado. Deseja-se encher a mochila obtendo o maior valor possível sem exceder a capacidade $c$. Formalmente o Problema da Mochila 0/1 pode assim ser definido:

$$
\begin{aligned}
& \max \sum_{j=1}^{n} p_{j} x_{j} \\
& \text { sujeito } a \sum_{j=1}^{n} w_{j} x_{j} \leq c, x_{j} \in\{0,1\}, \quad j=1, \ldots, n
\end{aligned}
$$

Onde $x_{j}$ é igual a 1 se o item $j$ está incluído na mochila e 0 caso contrário. Sem perda de generalidade assume-se que $w_{j} \leq c$ para assegurar que todos os itens cabem na mochila e para evitar soluções triviais.

O problema da mochila apresenta ainda várias aplicações práticas no mundo real, como por exemplo, [17]: carregamento de cargas, seleção de projetos, corte de peças, controle orçamentário entre outras.

\section{APLICAÇÃO DO PROBLEMA}

O problema de seleção de critérios de padrão de atendimento objetiva maximizar o número de itens a serem colocados em um porto com capacidade de investimento. Seja $x j$, com $j=1, . .40$; itens de critério padrão definido pela ANTAQ no relatório executivo, Cada item tem um grau de importância para o porto, e poderá pertencer ou não ao porto. 
Seja wj $\operatorname{com} j=1, \ldots, 40$, peso de importância atribuído a cada item, onde esse valor é atribuído dentro do conjunto $\{1 . .5\}$. Esses pesos definem a importância do item para o porto.

Seja pj com $j=1, . .40$, custo teórico (subjetivo) de implantação de cada item, onde o custo é atribuído do conjunto \{1..5\}. Esse custo define quão caro é a implantação do item.

Seja Fmax o valor máximo a ser atingido pela soma de todos os pesos de importância dos itens de critério padrão de atendimento. VIT é a quantidade mínima de itens necessários num porto, multiplicados pelos seus graus de importância.

As variáveis de decisão do problema são:

$$
\begin{gathered}
x_{j}=\{1, \quad \text { se o item } j \text { for selecionado para o Porto; } \\
0, \text { se o item não for }\}
\end{gathered}
$$

E a formulação para a Seleção de Critérios Padrão (SCP) é

$$
F_{\max }(x)=\operatorname{Max} \sum_{\mathrm{j}=1}^{40} p_{\mathrm{j}} x_{\mathrm{j}}
$$

Sujeito à

$$
\begin{aligned}
& \sum_{i=1}^{40} w_{j} x_{j} \leq V I T \\
& x_{j} \in\{0,1\}
\end{aligned}
$$

A função (3) está relacionada à maximização dos itens totais atribuídos a um porto, com suas importâncias atribuídas. Esse valor máximo poderá ser alcançado, em termos dos valores de custo de implantação de cada item igual ou inferior ao valor de investimento total disponível para os itens, é assegurada pela restrição (4). E por fim, a restrição (5) garante as variáveis binárias e integralidade.

\subsection{APLICAÇÃO DO PROBLEMA DA MOCHILA 0/1}

Partindo da modelagem inicial e dos valores de importância e custo atribuídos aos itens no artigo, chegamos a função objetivo $\operatorname{Fmax}(x)$ para nosso caso (6).

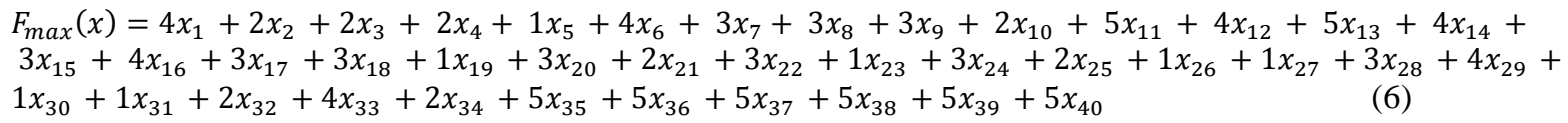

Sujeito à.:

$$
\begin{aligned}
& R(x)=5 x_{1}+4 x_{2}+4 x_{3}+3 x_{4}+2 x_{5}+3 x_{6}+3 x_{7}+3 x_{8}+3 x_{9}+2 x_{10}+4 x_{11}+4 x_{12}+5 x_{13}+3 x_{14}+ \\
& 1 x_{15}+2 x_{16}+2 x_{17}+3 x_{18}+2 x_{19}+1 x_{20}+2 x_{21}+3 x_{22}+3 x_{23}+3 x_{24}+3 x_{25}+3 x_{26}+3 x_{27}+2 x_{28}+3 x_{29}+ \\
& 2 x_{30}+1 x_{31}+2 x_{32}+3 x_{33}+2 x_{34}+5 x_{35}+5 x_{36}+5 x_{37}+4 x_{38}+4 x_{39}+4 x_{40} \leq \text { VTI (7) } \\
& \quad x_{j} \in\{0,1\}
\end{aligned}
$$

Para o presente estudo, analisaram-se três (3) cenários, onde VTI assume os valores de 40, 60 e 80. A equação (7), com VTI variável, para ser associado a cada cenário trabalhado nos resultados. Também definimos todos os valores inteiros e variáveis binárias $(0 / 1)$, conforme restrição representada pela equação (8).

Os resultados dos cenários foram comparados com cenários reais, avaliados pela ANTAQ [4], para os portos Roadway e Manaus Moderna, representando o melhor cenário atual (Roadway) e o cenário médio entre os portos regionais (Manaus Moderna). 


\section{RESULTADOS}

Observa-se, na Figura 2, que todos os cenários apresentam melhorias significativas na qualidade do padrão de atendimento, quando comparados com o cenário médio. Neste caso, o resultado aumenta o padrão de atendimento em média $90 \%$. Entretanto, observa-se que o porto Roadway apresenta um desempenho elevado, ficando $6,6 \%$ abaixo apenas do cenário (80).

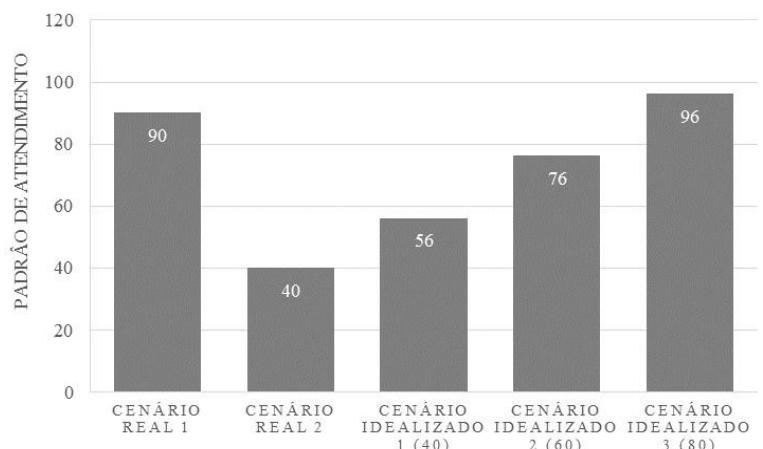

Figura 2 - Padrão de Atendimento nos portos de Manaus

Fonte: Os autores

A Tabela 1 mostra os resultados da execução para os cenários (40), (60) e (80). Observa-se que os cenários selecionaram uma quantidade de itens maiores em $27 \%, 63 \%$ e $127 \%$, respectivamente, que o cenário médio. Entretanto, destaca-se que na solução ótima para ambos a seleção dos itens repetia a importância dos critérios e não prejudica o objetivo do porto.

Tabela 1 - Itens selecionados pelo modelo proposto.

\begin{tabular}{|c|c|c|c|}
\hline Cenário & Itens de Padrão de Atendimento & $\begin{array}{l}\text { Qtd. de itens } \\
\text { selecionados }\end{array}$ & $\begin{array}{l}\text { Comparação com } \\
\text { o cenário médio }\end{array}$ \\
\hline $\begin{array}{l}\text { Cenário } \\
\text { Idealizado } \\
\text { (40) }\end{array}$ & $\begin{array}{c}\text { Área compatível com a demanda de passageiros no terminal; Serviço } \\
\text { de abastecimento de água para embarcações; Serviço de combate a } \\
\text { incêndio; Salas de administração e arrecadação; Balcão de } \\
\text { informações;Boxe de vendas de passagens;Bancos/assentos; } \\
\text { Lixeiras; Bancos e assentos; Banheiros: masculinos e femoninos; } \\
\text { Quadro de chegada e saída de embarcações; Berço específico e } \\
\text { adequado para embarque e desembarque de passageiros; Suficiência } \\
\text { de berços; Berço compatível com as características da embarcação } \\
\text { operante; berço compatível com as características da embarcação } \\
\text { operante; }\end{array}$ & 14 & $27 \%$ Acima \\
\hline $\begin{array}{l}\text { Cenário } \\
\text { Idealizado } \\
(60)\end{array}$ & $\begin{array}{l}\text { Área compatível com a demanda de passageiros no terminal; Serviço } \\
\text { de abastecimento de água para embarcações; Serviço de combate a } \\
\text { incêndio; Abastecimento de energia; Salas de administração e } \\
\text { arrecadação; Balcão de informações; Boxe de vendas de passagens; } \\
\text { Bancos/assentos; Lixeiras; Bancos e assentos; Banheiros: masculinos } \\
\text { e femoninos; Quadro de chegada e saída de embarcações; Berço } \\
\text { específico e adequado para embarque e desembarque de passageiros; } \\
\text { Suficiência de berços; }\end{array}$ & 18 & $63 \%$ Acima \\
\hline $\begin{array}{l}\text { Cenário } \\
\text { Idealizado } \\
(80)\end{array}$ & $\begin{array}{l}\text { Área compatível com a demanda de passageiros no terminal; Guarita } \\
\text { de controle; Posto de atendimento médico;Posto de polícia;Serviços } \\
\text { de carregadores; Serviço de combate a incêndio; Abastecimento de } \\
\text { energia; Salas de administração e arrecadação;Balcão de } \\
\text { informações; Boxe de vendas de passagens; Bancos/assentos; } \\
\text { Banheiros públicos: masculino/feminino; Lixeiras; Quadro de } \\
\text { chegada e saídas das embarcações; Policiamento; Bancos e assentos; } \\
\text { Banheiros: masculinos e femoninos; Quadro de chegada e saída de } \\
\text { embarcações; Berço específico e adequado para embarque e } \\
\text { desembarque de passageiros; Suficiência de berços; berço compatível } \\
\text { com as características da embarcação operante; }\end{array}$ & 25 & $127 \%$ Acima \\
\hline
\end{tabular}

Fonte: Os autores 


\section{CONSIDERAÇÕES FINAIS}

A proposição do Problema da Mochila 0/1 para definição do padrão de atendimento mostra-se válido no sentido de selecionar os itens mais importantes para operação portuária. Para o caso aqui apresentado, verificou-se que os cenários (40), (60) e (80) são mais eficientes que os atuais e podem ser utilizados com referência (benchmarks) para que portos considerados ineficientes melhorem seus desempenhos.

O resultado da aplicação da metodologia apresentada pode proporcionar uma melhoria do transporte fluvial na Amazônia. A aplicação do modelo proposto oferece um diagnóstico de onde deve ser melhorado, influencia no planejamento das políticas públicas, na regulamentação, qualificação e especialização do setor, além de fornecer alinhamento conceitual aos usuários.

Quanto à contribuição deste trabalho, espera-se que, por meio da utilização do Problema da Mochila 0/1, os tomadores de decisão e usuários possam tomar decisões mais precisas, tais como: Quais itens de padrão de atendimento devem ser selecionados para um bom funcionamento de um porto?

Como proposta para futuras pesquisas, sugere-se o desenvolvimento de um cenário para ETC, IP4 e TUP uma vez que, estes portos são responsáveis por realizar objetivos diferentes. Assim, o modelo proposto poderia refletir melhor as condições de todo sistema portuário que está associado com o transporte hidroviário.

\section{AGRADECIMENTOS}

Os autores agradecem o apoio do SINDARMA e do CNPq. Também, agradecem ao INTRA - Instituto de Pesquisa em Transportes e ao Laboratório TRANSPORTAR pelo suporte aos membros envolvidos no desenvolvimento do artigo.

\section{REFERÊNCIAS BIBLIOGRÁFICAS}

[1] Agência Nacional de Transportes Aquaviários - ANTAQ (2009) Regulamentação das Instalações Portuárias Públicas de Pequeno Porte - IP4. 〈http://www.antaq.gov.br〉. Acessado em 21 de Janeiro de 2015.

[2] Agência Nacional de Transportes Aquaviários - ANTAQ (2009). Seminário internacional sobre hidrovias. Brasília - DF. Disponível em <http://www.antaq.gov.br/Portal/Palestras.asp >. Acessado em 21 de Janeiro de 2015.

[3] Agência Nacional de Transportes Aquaviários - ANTAQ (2009). Termos e conceitos Técnicos - Anuário portuário. Disponível em: 〈http://www.antaq.gov.br>. Acessado $\begin{array}{lllll}\text { em } & 21 & \text { de } & \text { Janeiro }\end{array}$ 
[4] Agência Nacional de Transportes Aquaviários - ANTAQ (2013) Caracterização da oferta e da demanda do transporte fluvial de passageiros da região amazônica - Brasília: 108p.: il.

[5] BUSSINGER, F. Reformas e regulação portuária. Rio de Janeiro: IPEA.

[6] Confederação Nacional do Transporte - CNT (2011). O entrave portuário no Brasil. Economia em foco. Brasília.

[7] DEGRASSI, S. (2001). The seaport network Hamburg. 2001. Tese (Doutorado) Universidade de Hamburgo, Hamburgo.

[8] Instituto de Pesquisa Econômica Aplicada - IPEA (2009). Portos brasileiros 2009: Ranking, área de influência, porte e valor agregado médio dos produtos movimentados. Texto para Discussão n. 1408.

[9] Instituto de Pesquisa Econômica Aplicada - IPEA (2009). Texto para discussão $n^{\circ}$

1423. Gargalos e demandas da infraestrutura portuária e os investimentos do PAC: Mapeamento IPEA de obras portuárias. Brasília.

[10] KRASNOGOR, N. (2002) Studies on the theory and design space of memetic algorithms. Faculty of computing, engineering and mathematical sciences. University of the West of England, UK. 2002, PhD thesis.

[11] Maria Helena Macdowell Barbosa. (1982). Diretrizes para Projetos de Terminais Hidroviários Urbanos de Passageiros. 1982. Tese (Doutorado) - Instituto Militar de Engenharia - IME, Rio de Janeiro.

[12] MARTELLO, S.; P. Toth (1977). An upper bound for the zero-one knapsack problem and a branch and bound algorithm. European Journal of Operational Research, 1: 169175.

[13] MARTELlO, S.; P. Toth (1988). A new algorithm for the 0-1 knapsack problem. Management Science, 34(5): 633-644.

[14] MORAES, H. B. (2003) Portos. Material Didático. Departamento de Engenharia de Transportes, Centro Tecnológico. Universidade Federal do Pará, p. 2-7.

[15] PISINGER, D. (1993). "An expanding-core algorithm for exact 0-1 Knapsack Problem”. European Journal of Operational Research, 87:175- 187, 1995.

[16] RODRIGUES, P. R. A. (2007) Introdução aos sistemas de Transporte no Brasil e à logística internacional, 4 edição. 4. ed. São Paulo: Aduaneiras, v. 1.

[17] KARP, Richard M. (1972). "Reducibility Among Combinatorial Problems". In R. E. Miller and J. W. Thatcher (editors). Complexity of Computer Computations. New York: Plenum. pp. 85-103. 